\title{
A VLA STUDY OF OOTY SMALL DIAMETER SOURCES
}

\author{
T.K.MENON \\ Department of Geophysics and Astronomy \\ University of British Columbia \\ Vancouver, B.C. V6T 1Z4, CANADA
}

\section{Introduction}

In the Ooty lunar occultation survey of about 711 sources at $327 \mathrm{MHz}$ over 200 sources were classified as unresolved with upper limits of about 4" (Kapahi(1986)). In order to study the nature of these sources I have observed a sub-sample of 71 sources with the VLA in the A-Configuration at $6 \mathrm{~cm}$. The angular resolution ranged from 0.4 to 0.8 arc second. My observations show that of the above 71 sources $56 \%$ are well resolved doubles and triples resembling FRII sources with angular separations of $0^{\prime \prime} .4$ to $8^{\prime \prime}$. The flux density ratios of well separated doubles and triples range from 1 to about 8 . However the LAS of sources with ratios of less than 2 is found to increase with decreasing flux density. Since most of the low flux density sources are in blank fields in the PSS prints no redshift information is available at present. Being FRII sources they are likely to be very distant sources at comologically interesting ranges of redshift. In order to test whether the above observed increase in the angular size is of cosmological significance we need optical identification and redshift information. These are being planned.

The study has provided detailed maps of a large number of extended sources with sizes less than about $4^{\prime \prime}$. This has led to the discovery of 4 gravitational lens candidates among the above sources. One of the most interesting is a ring structure shown in Fig. 1. The basic source is a typical triple source having a total flux density of about $50.9 \mathrm{mJy}$ at $6 \mathrm{~cm}$ and with about $12 \%$ of the total flux density in a central component. As is seen from Fig. 1 there is a spectacular arc structure extending westward from the main source. In addition it is seen that the contours are also distorted with a noticeable hole on the eastern side suggesting that the arc may indeed be a more complete structure but for the presence of the main source. 

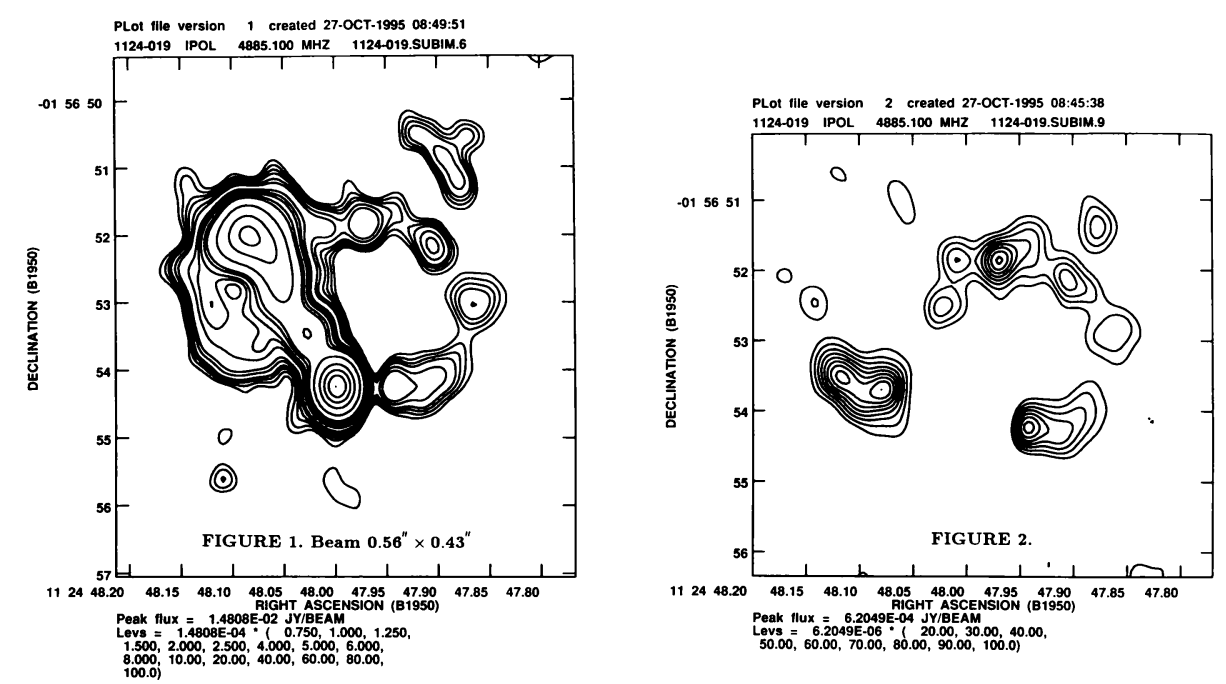

The image of the residual structure, after an approximate subtraction of the main source, shown in Fig. 2 resembles a ring of emission of diameter $3^{\prime \prime}$ with a number of hot spots on the periphery and a bright source at it's eastern end. In the presently known cases of radio ring systems such as MG1131+0456 or $1549+307$ (Schneider et al(1992)) the components of an extended background source are suggested to have been imaged by a foreground galaxy into rings and multiple images. However the geometrical situation in the present case appears to be more complex. If the triple source is a background source it is seen as almost undistorted and the center of the ring itself is displaced to the west of the central component of the triple source. Hence it is not clear at present whether the whole image is a superposition of two independent systems one of which is a gravitationally imaged system or the two systems are related by gravitational lensing process. The suggested optical identification for the source is an object which is about 1.5 arc second displaced from the central component towards the southwest. There is at present no information on any other optical object in the vicinity of the radio source. Deeper optical data are being obtained to clarify the situation.

These investigations were supported by a grant from the Natural Sciences and Engineering Council of Canada. National Radio Astronomy Observatory is operated by Associated Universities, Inc., under cooperative agreement with the National Science Foundation.

\section{References}

Kapahi, V. 1986, Proc. I.A.U. Symp. 124, A.Hewitt, G. Burbidge, \& L.Z. Fang (Eds.), Dordrecht: Reidel, p251

Schneider, P; Ehlers, J; Falco, E.E, 1992, " Gravitational Lensing ", (Springer-Verlag. Berlin) 